\title{
AFRONTAMIENTO DEL ESTRÉS EN FAMILIAS CUYO PADRE HA EMIGRADO A LOS ESTADOS UNIDOS: UN ESTUDIO DE REESTRUCTURACIÓN FAMILIAR Y RESPONSABILIDADES FILIALES
}

\author{
COPING STRATEGIES OF FAMILIES WHOSE FATHER HAS MIGRATED TO THE \\ UNITED STATES OF AMERICA: \\ A STUDY OF FAMILY RE-STRUCTURING AND FILIAL RESPONSIBILITIES*
}

BERNARDO ENRIQUE ROQUE TOVAR**

INSTITUTO TECNOLÓGICO Y DE ESTUDIOS SUPERIORES DE OCCIDENTE (ITESO), GUADALAJARA - MÉXICO

MARTHA GABRIELA RAMÍREZ GONZÁLEZ***

TERAPEUTA SISTEMA PARA El DESARROLLO INTEGRAL DE LA FAMILIA (DIF), ZAPOPAN - MÉXICO

FECHA RECEPCIÓN: 25/10/2012 • FECHA ACEPTACIÓN: 8/2/2013

\begin{abstract}
Resumen
El presente artículo se centra en el estudio de familias cuyo padre emigra a los Estados Unidos de América (EUA), dejando a su esposa e hijos en México, algunas veces con la esperanza de reunirse eventualmente o en otras con la claridad de que vivirá una temporada «aquí» y otra «allá», una pauta que ha sido descrita ampliamente por autores como Suárez-Orozco, Todorova y Louie (2002) y denominada como «pérdida ambigua» (Boss, 2001). El objetivo general del estudio fue describir las estrategias de afrontamiento de los miembros de familias cuyo padre emigra a los Estados Unidos por períodos prolongados. Se utilizó un enfoque cualitativo para estudiar el fenómeno en una comunidad de Zapopan, Jalisco, México. Se describen los recursos de afrontamiento de estas familias durante la separación, así como los conceptos de parentalización y responsabilidad filial, que están insertos en el «estrés relacional» que implica el fenómeno de la migración. La teoría de la Terapia Familiar Estructural también sirvió de base para demostrar cómo los miembros de una familia con el padre emigrado logran mantener la imagen/autoridad paterna ante su ausencia, además de que se encontró la existencia de un apoyo de la familia extensa ante la ausencia del padre. Finalmente se observó una comunicación continua y significativa del padre con su familia como una estrategia de afrontamiento eficaz.
\end{abstract}

* Los autores quieren agradecer a la Dra. Liliana Lira y al Dr. Rodrigo González por su asesoría metodológica y temática para llevar esta investigación a cabo. Además, se agradece a la Dirección General del Sistema Desarrollo Integral de la Familia (DIF) en Zapopan, Jal. México, por haber autorizado la localización de los participantes de este estudio a través de sus Centros de Desarrollo Comunitario.

** Instituto Tecnológico y de Estudios Superiores de Occidente, Departamento de Salud, Psicología y Comunidad. Maestro en terapia familiar sistémica y Master of Science - Professional Counseling. Periférico Sur Manuel Gómez Morín 8585. CP 45604 Tlaquepaque, Jal. México. Departamento de Salud, Psicología y Comunidad. Tel. 3669-3434 ext. 3215. bernardoroque@iteso.mx

*** Sistema para el Desarrollo Integral de la Familia DIF Zapopan Centro de Desarrollo Comunitario Agua Blanca. Maestro en terapia familiar sistémica. Centro de Desarrollo Comunitario n. ${ }^{\circ} 9$ DIF Zapopan. Álvaro Obregón 187 Col. Agua Blanca Industrial, Zapopan Jal. México. CP 45235 Tel. 36867932. pgrmz@yahoo.com 
Se concluye que los miembros de la familia que se quedan en el país de origen ven a la ambigüedad (estar/no estar), como el principal factor a afrontar durante la separación.

Palabras clave: migración internacional, composición familiar, estrés psicológico, adaptación psicológica.

\begin{abstract}
The present article is focused on the study of families whose father has migrated to the United States of America (USA), leaving his wife and children in Mexico, sometimes with the hope of meeting all together or in other times with the clear idea that the father will go back and forth, sometimes «here» and sometimes «there». This pattern has been widely described for authors such as Suárez-Orozco, Todorova and Louie (2002) and labeled as an «ambiguous loss» (Boss, 2001). The main objective of this research was to describe the coping strategies of family members whose father migrates to the USA by extended periods of time. A qualitative research approach was used to study this phenomenon in a community at Zapopan, Jalisco, Mexico. The coping strategies of these families during separation are described, along with the concepts of parentification and filial responsibility, which are inserted within the «relational stress» that migration implies. The theory of Structural Family Therapy is also used to show how these families are able to maintain the paternal image and authority, despite his absence. It was also noted a supportive role played by the extended family when the father was lacking and also a continuous and meaningful communication between the migrant father and his family as a coping strategy for this process. It was concluded that the family members that stay at the country of origin look at the ambiguity (being present/being absent) as the main element to cope with during separation.

Keywords: emigration and immigration, family characteristics, psychological stress, psychological adaptation.
\end{abstract}

\section{Introducción}

La migración de ciudadanos mexicanos hacia los Estados Unidos de América (EUA) es un fenómeno bastante estudiado y de gran importancia económica, social y política para el país. Entre los muchos aspectos que son posibles de estudiar de este fenómeno está el de las consecuencias en la estructura familiar que conlleva para muchas familias. Así, según cifras disponibles del Instituto Nacional de Estadística, Geografía e Informática (INEGI) en la década pasada (1990 al 2000) emigraron a los EUA un total de 3'237.841 personas mexicanas, de las cuales 2'336.447 fueron hombres, y el resto mujeres. Es decir, el $72 \%$ del total de emigrantes eran de sexo masculino (INEGI, 2004). Cifras más recientes, ubican en 485.000 aproximadamente la cifra de personas que anualmente abandonan el país hacia los EUA en busca de mejores condiciones de vida (Orozco \& Sarabia, 2005). Tomando en consideración estas cifras, es posible entender que muchos de estos varones dejan a su familia en México, mientras ellos laboran en el otro país, trayendo como consecuencia importantes cambios en la estructura, roles y funciones que la familia debe de ejercer para su funcionamiento.

En la familia del migrante que se queda en el país de origen, no es infrecuente el que los hijos asuman responsabilidades que hubieran sido cubiertas con la presencia del padre, tales como proveer de soporte emocional a la mamá y a otros hermanos, ayudar en la economía familiar e interactuar con la escuela y la comunidad a nombre de la familia. De esta forma, es posible pensar que uno o más de los hijos que se quedan en el país de origen pueden experimentar niveles de estrés (expresados tanto en actividades como en sentimientos de lealtad) que pudieran no ser experimentados por otros niños o jóvenes de su edad en familias no migratorias. Por otro lado, la esposa-madre se puede ver ante una situación de cierta pérdida ambigua, por la ausencia de su esposo, y ante una situación potencialmente estresante, ya que queda a cargo de los hijos y de la familia en general. Por último, el padre emigrante emprende un proceso que en la mayoría de los casos tiene como objetivo final el avance económico y social de la familia que ha dejado en su lugar de origen. Visto este fenómeno desde la teoría transaccional del estrés, (Lazarus, 1991; Matheny \& McCarthy, 2000), este proceso podría ser considerado como el resultado de un desbalance entre las demandas percibidas y los recursos de afrontamiento reconocidos.

La investigación tiene relevancia social si se considera que la cifra de mexicanos que emigran a los EUA año con año que han sido mostradas previamente. Tal aumento en la comunidad mexicana del citado país hace que los trabajadores de la salud mental necesiten conocer acerca de las diferencias culturales que tienen las familias de origen latino en relación a las familias anglosajonas. Así, algunos autores como Falicov (2007), han llamado la atención sobre este fenómeno y la im- 
portancia de entender el sentido de familia de los «inmigrantes trans-nacionales» para ofrecer mejores servicios terapéuticos a esta población. Esta misma autora también ha acuñado el término «estrés relacional», el cual utiliza para referirse a toda la serie de separaciones, reunificaciones y nuevas separaciones que la migración conlleva. En sus palabras, «la migración siempre involucra separación» (Falicov, 2007, p. 160). Por lo tanto, para el terapeuta que trabaja en EUA con poblaciones de origen latino y sobre todo de origen mexicano, los resultados de esta investigación le informarán acerca de aspectos psicosociales a considerar en su práctica clínica. De manera más relevante, la investigación aporta al campo teórico-conceptual de las explicaciones que fundamentan el fenómeno migratorio. Por otro lado, el valor teórico de esta investigación se puede entender desde qué tipo de teoría es más útil al acercarse a este objeto de investigación. Efectivamente, el fenómeno de asumir responsabilidades paternas ha sido descrito con el término de parentalización, por el trabajo de Boszormeny-Nagy (1973), y aunque el mismo autor advierte que no siempre se trata de una situación desventajosa sino adaptativa, el término ha tenido un matiz más bien patológico, sobre todo si es visto desde la óptica de las culturas donde la migración es necesaria para la supervivencia misma. Desde una perspectiva más ecosistémica y menos clínica, autores como Jurkovic, Kuperminc, Perilla, Murphy, Ibañez y Casey (2004) han desarrollado el término responsabilidad filial, con énfasis en el familismo y en la justicia de las responsabilidades que son asignadas a los hijos que pertenecen a la familia del padre migrante, en cuanto a tareas de apoyo instrumental, tales como el ayudar en las tareas domésticas del hogar y el brindar apoyo emocional a la madre y hermanos. Es en esta perspectiva en la que se inserta esta investigación.

En el mismo sentido, en un estudio anterior Roque, Kuperminc, Ramírez y Álvarez (2009), se confirmó que uno de los principales motivadores de los padres para tomar la decisión de emigrar es el futuro económico y educativo de los hijos, y en ese contexto se entiende de una manera diferente la reciprocidad y justicia que puede existir en los cambios estructurales que conlleva este proceso.

Aun cuando la teoría de la parentalización y la responsabilidad filial no son excluyentes, sino más bien parte de un mismo espectro continuo, el presente trabajo trató de comparar ambas perspectivas teóricas con referencia a la percepción y manejo del estrés, con el objetivo de refinar la aplicación de ellas en los hijos de la familia del migrante.

De esta manera, es posible pensar que la estructura de las familias con un miembro migrante experimentan cambios importantes como producto de tal fenómeno. Uno de los autores que más ampliamente ha desarrollado el concepto de «estructura familiar» en la historia de la terapia familiar es Salvador Minuchin y sus colaboradores. Esta teoría tiene que ver con la existencia de límites (rígidos o difusos) que existen entre los diferentes subsistemas (holones) que integran a la familia. La palabra «holón» es un término que hace referencia tanto a la parte como al todo. El autor distingue tres «holones» principales:

Conyugal: Formado por los esposos, dos adultos unidos con la idea de formar una familia.

Parental: EL susbsistema conyugal más por lo menos un hijo.

Fraterno: La interacción que se da solo entre los hermanos (Minuchin, 1974).

La organización que adquiera el funcionamiento de la familia con respecto a sus límites y roles, conformará una estructura típica, que hará frente a las dificultades de las tareas de la familia, o dificultará su crecimiento. En este punto, una de las fuentes de tensión y/o estrés que señala el autor tiene que ver con las dificultades extrafamiliares de los miembros de la familia como un todo. El caso de un proceso migratorio estaría ubicado en tal categoría.

La teoría conlleva de manera implícita una idea de autoridad de tipo vertical, donde es el holón conyugal el que decide los roles, jerarquías, límites y fronteras entre los holones y en las interacciones de la familia con otros sistemas.

Como se puede apreciar, estas ideas resultan de interés para ser utilizadas en el estudio de procesos de separación inducidos por la migración. Si aceptamos la idea de autoridad vertical que plantea este autor, entonces es interesante hacerse preguntas tales como: ¿qué sucede con la autoridad del padre cuando se emigra? ¿Cómo logra mantenerla? ¿Qué transacciones se dan con la familia extensa tanto de la madre como del padre? ¿Cómo lograr 
una frontera para seguirse diferenciando pero al mismo tiempo tener la flexibilidad suficiente para recibir el apoyo que es normal que las familias extensas proporcionen cuando no se encuentra presente el padre de familia? ¿Qué sucede con el holón fraterno? ¿Cómo apoyan a la mamá sin pasar la jerarquía del padre? ¿Cómo podrían ser hijos y apoyo de la mamá al mismo tiempo? ¿Cómo diferenciar esos roles?

La teoría no está exenta de sus críticos, sobre todo en autores de corte feminista (v. g. Becvar \& Becvar, 1996). Específicamente, la crítica va en el sentido de que la autoridad vertical deja todo el poder en manos de los padres (holón parental) y eventualmente a la madre se le pueden achacar muchas de las disfuncionalidades de la familia. Aun así, se considera que la teoría estructural de Minuchin representa un muy buen marco de referencia para el estudio.

Por otro lado, otro de los apoyos teóricos para la interpretación de resultados de este estudio es la anteriormente mencionada teoría transaccional del estrés. Tal teoría señala que el estrés es el resultado de un desbalance entre una situación específica y los recursos con los que cuenta la persona (o el sistema) para afrontarlo (Lazarus \& Folkman, 1984). En el caso de la migración, el fenómeno presenta puntos interesantes si es analizado bajo esta teoría, ya que para el papá que emigra, el tipo de estrés está más relacionado al hecho de que representa una situación de ajuste a un nuevo contexto social. En cambio, para la familia que se queda en el país de origen, se asocia más a que la situación a afrontar tiene que ver con una serie de reunificaciones y cambios en la configuración familiar que seguirán cierto patrón cíclico, a menos que toda la familia desee reunirse con el padre a donde este emigró.

El mismo autor menciona que existe la tendencia a creer que el mejor estrés es el que lleva a la persona a un estado evolutivo superior, es decir, que puede ser resuelto y manejado (muy en el sentido darwiniano de la selección de las especies). No obstante, Lazarus (1984) aclara que esto está basado en un valor occidental e individualista, ya que existen eventos que por su propia naturaleza no son tan fáciles de ser «superados». Por lo tanto, en este trabajo es muy importante aclarar que el término «afrontamiento» no necesariamente implica una «solución adaptativa» a la separación que produce la emigración, sino un proceso basado en la falta de certeza y que produce que las familias saquen los mejores recursos con los que cuentan para «hacer frente» a sus reorganizaciones familiares y a mantener el sentido de familia aún en la distancia, pero algunos recursos serán más efectivos que otros.

Las limitaciones pueden ser reales o irreales, lo que importa es la medida en que permiten o impiden acciones para afrontar una situación estresante. Por ejemplo, un adolescente cuyo padre ha emigrado a los EUA, pudiera pensar que no es adecuado contarle sus problemas a su mamá, porque su papá le dijo que en su ausencia él sería «el hombrecito de la casa».

Así, la presente investigación tuvo como objetivo general describir las estrategias de afrontamiento de los miembros de familias cuyo padre emigra a los Estados Unidos por períodos prolongados.

\section{Método}

Esta investigación se realizó bajo un enfoque cualitativo. Se empleó el método de las historias de vida temáticas. Tal y como es explicado por Bolívar, Domingo y Fernández (2001), en el presente estudio se retomó el sentido del término como life story, es decir un relato de vida. La característica principal, según este autor, es que se trata de una «narración (retrospectiva) por el propio protagonista de vida o de determinados fragmentos/aspectos de ella, por iniciativa propia o por requerimiento de uno o varios interlocutores» (p. 28). Para el caso de esta investigación, se retomó lo relativo a la narración que los participantes puedan proporcionar acerca del tema a investigar. Se trata de un tratamiento temático de sus vivencias con la migración de los padres y la forma como la han afrontado y no de la vida de los participantes en general.

Este método tiene su fundamento epistemológico en la fenomenología., entendiendo a esta última como la posibilidad de «considerar el mundo en el que vivimos intuitivamente, con sus realidades, pero tal y como se nos presente en primer término en la experiencia lisa y llana y también en las maneras en que caen a menudo en la fluctuación por lo que hace a su validez» (Bolívar, Domingo \& Fernández, 2001).

\section{Instrumento de recolección de datos}

Para esta investigación se utilizaron entrevistas a profundidad semiestructuradas, las cuales permiten ac- 
ceder a las perspectivas construidas a partir de las experiencias de vida de los participantes. Se diseñó una guía de preguntas que exploran el tema de investigación. Partiendo de las sugerencias de Patton (1987) respecto al tipo de preguntas que se pueden realizar en una entrevista semiestructurada, se diseñaron preguntas que principalmente inquieren acerca de las experiencias de los participantes respecto al tema de investigación. Las preguntas de experiencia, según el autor referido, inquieren acerca de las vivencias concretas de las personas entrevistadas, y se recuperan sus pensamientos, creencias, conceptos y valoraciones. Aunque las preguntas experienciales pueden generar estados afectivos y emocionales, se dejó que estos fueran expresados libremente, más que dirigirnos directamente hacia ellos.

\section{Participantes}

Para la selección de los participantes, se llevó a cabo la elección por conveniencia (Hernández, Fernández \& Baptista, 2006). El objetivo principal de este tipo de selección es el elegir a las personas a investigar bajo ciertos criterios que son los que interesan al investigador. Para el presente estudio, se recogió una muestra conformada por 7 familias con adolescentes entre los 12 y los 17 años de edad, los cuales estaban cursando el nivel de educación secundaria y en donde el padre de estas familias emigró a los EUA por lo menos durante un año, del 2007 al 2010. Dicha muestra se obtuvo por medio de los encargados de asesoría psicológica de una institución gubernamental en el Zapopan, Jalisco, México, la cual se dedica al desarrollo familiar y comunitario.

\section{Análisis de resultados}

Respecto a análisis de resultados, se utilizó la triangulación de investigadores y la saturación teórica, tal y como es descrita por Hernández, Fernández y Baptista (2006). Este método de análisis consiste, principalmente, en la lectura recurrente de las transcripciones obtenidas durante las entrevistas, de manera tal que van emergiendo códigos y categorías relacionadas con el objetivo de la investigación. Posteriormente, cada uno de los investigadores expone la categorización encontrada con el fin de obtener un consenso entre los investigadores, lo cual se denomina triangulación de datos, descrito por los autores.
Antes de realizar la entrevista, se les pidió a los participantes su autorización a través de un formato de consentimiento informado, el cual señalaba aspectos éticos tales como el anonimato de la identidad, el objetivo del estudio, la posibilidad de retirarse de la entrevista en cualquier momento y el derecho a no contestar algunas de las preguntas que les fueron formuladas.

Los entrevistados están señalados con letras para mantener su anonimato. El investigador es identificado con la letra «E» de «Entrevistador». Las entrevistas se llevaron a cabo entre los meses de enero a junio de 2010 y a todos los participantes se les pidió su consentimiento informado para participar de la investigación.

\section{Resultados}

A continuación se presentan algunos ejemplos de los resultados obtenidos durante la realización de las entrevistas a profundidad que fueron hechas a los participantes. Se identificaron tres núcleos temáticos relevantes que en la interpretación de los investigadores explican formas de afrontamiento familiar donde el padre ha decidido emigrar a los EUA, quedándose esposa e hijos en el país de origen. Los núcleos temáticos muestran tres «miradas» distintas para explicar el fenómeno, y en donde algunos de los procesos específicos encontrados pueden superponerse en más de uno de los núcleos.

En la tabla 1 se esquematizan estos tres núcleos temáticos. El título superior en cada columna se refiere al nombre del núcleo, y los tres o cuatro títulos debajo de cada uno de ellos se refieren a los códigos encontrados en cada núcleo, dentro del relato de cado uno de los entrevistados.

El primero de ellos ha sido denominado Mantenimiento de la imagen/autoridad paterna ante su ausencia. Este núcleo describe el proceso mediante el cual la familia mantiene la importancia del papá en las decisiones de crianza y educación de los hijos, a pesar de la distancia. El segundo se denomina Apoyo/re-acoplamiento familia extensa ante la ausencia paterna, el cual explica las reacciones que la familia extensa (tanto política como consanguínea) tiene con la mamá y los hijos del padre migrante cuando este se ausenta. Por último, el núcleo Comunicación continua y significativa del padre con su familia, tiene que ver con la comunicación sincrónica y asincrónica que tanto 
el padre como la familia generan durante los períodos de ausencia, que se vuelve un mecanismo eficaz para afron- tar la separación. Para una mejor comprensión de estos núcleos temáticos, se presenta la tabla 1:

Tabla 1. Resultados del análisis cualitativo: núcleos temáticos.

\begin{tabular}{ll}
\hline \hline $\begin{array}{l}\text { Núcleo temático 1: } \\
\text { mantenimiento de la imagen/ } \\
\text { autoridad paterna ante su ausencia }\end{array}$ & $\begin{array}{l}\text { Núcleo temático 2: } \\
\text { Apoyo/re-acoplamiento familia } \\
\text { extensa ante la ausencia paterna }\end{array}$ \\
\hline $\begin{array}{l}\text { Instauración de la imagen paterna a } \\
\text { través de actos significativos. }\end{array}$ & $\begin{array}{l}\text { Postura ante la familia de origen de } \\
\text { la esposa. }\end{array}$ \\
$\begin{array}{l}\text { Respeto del holón conyugal en la } \\
\text { distancia y ausencia. }\end{array}$ & $\begin{array}{l}\text { Postura ante la familia de origen del } \\
\text { esposo emigrado. }\end{array}$ \\
$\begin{array}{l}\text { Mantenimiento ante las familias de } \\
\text { origen. }\end{array}$ & $\begin{array}{l}\text { Permeabilidad de la frontera familiar } \\
\text { nuclear y extensa - Apoyo mutuo y } \\
\text { respeto de los sistemas. }\end{array}$ \\
& $\begin{array}{l}\text { Perspectivas a futuro de la familia de } \\
\text { origen ante el eventual retorno del } \\
\text { padre emigrado. }\end{array}$ \\
\end{tabular}

Núcleo temático 3:

Comunicación continua y significativa del padre con su familia

Uso de tecnología - Diferencias y similitudes culturales.

La «llamada» como ritual familiar.

Efectividad del medio comunicativo en la toma de decisiones familiares.
El primer núcleo temático describe el proceso mediante el cual la familia mantiene la importancia del papá en las decisiones de crianza y educación de los hijos, a pesar de la distancia. Este proceso se articula principalmente en el holón parental para después ser proyectado al holón de los hijos, hacia las familias extensas y hacia otras relaciones significativas. Tiene que ver con el establecimiento de una frontera familiar y de reglas y límites para los hijos. Por ejemplo, obsérvese cómo la mamá transmite al hijo la percepción de que tiene papá aún en la distancia:

Mamá 1: Ya estaba R en el kínder, cuando yo eché de ver que le afectó, hicieron un... pescado grandote para el papa y los niños le dijeron «ah! Pero R no tiene papá ¿a quién se lo va a dar?» $\mathrm{Y}$ todos los niños y un amigo le dijo, su amigo J de A a $\mathrm{R}$ «pos se lo das a mi papá», y yo fui por $\mathrm{R}$ al kínder, $\mathrm{R}$ iba con su pescadote de cartulina y me dice $\mathrm{R}$, «verdad mamá que sí tengo papá» «sí, ¿por qué mijo?» y ya R se agarra llorando y me agarré llorando yo (empieza a llorar) y mis amigas, y la maestra del kínder de $\mathrm{R}$ era psicóloga, ella me ayudaba mucho con $\mathrm{R}$ porque R era puro llorar y él quería a su papá y cuando él estuvo en segundo de kínder, lo tenía en el kínder de Winni Pooh y lo llevaba mi esposo en una moto diario, diario, él lo llevaba al kínder y yo lo recogía, entonces así quedó R lo recordó más porque en el kínder si hablan más de los papás, háganle un regalo a sus papás, ya cuando R estuvo en primero su papá llegó del norte o sea duró más de un año sin él».

Este mantenimiento de la figura del padre pareciera ser de gran relevancia para la cohesión y la estructura de las funciones parentales. En cierta medida, los conceptos desarrollados por Minuchin (1974), son pertinentes para entender este fenómeno. Por ejemplo, el concepto de holón (sistema y subsistema a la vez), permite comprender que para esta madre es importante hacer entender al hijo que su papá sigue «presente» tanto como su esposo (Holón conyugal) así como en sus funciones paternas (holón parental). Esta reorganización de la familia se puede concebir como una de las tareas principales para que los miembros conserven su sentido de pertenencia.

En otra parte de los relatos, dos adolescentes entrevistados por separado, reconocen la importancia de la figura del padre, no solo para ellos, sino también para su mamá:

E.- ¿Cómo sería tu vida si tu papá estuviera aquí?

P.- pues diferente

E.- ¿Cómo te la imaginas?

P.- iAy pues no sé en realidad no me alcanzo a imaginar porque son 8 años, yo tenía 5 años cuando se fue... 
entonces no me imagino plenamente como pudiera ser, pero yo pienso que todo sería mejor.

$$
\begin{aligned}
& \text { E.- ¿Sí? } \\
& \text { P-- Sí } \\
& \text { E.- ¿En qué sentido? }
\end{aligned}
$$

P.- Sentimentalmente igual y a lo mejor sí, porque pues sí me hace mucha falta, y pues a mi mamá también le hace falta.

E.- Es interesante porque a pesar de que tu papá está afuera sigue estando presente entre ustedes.

A.- Pienso que de todos modos sería importante que él estuviera... por una parte mi mamá se sentiría mejor... yo pienso que era necesario tener que vivir esto, a pesar de que por una parte lo resentimos mucho, yo pienso que fue bueno.

En las preguntas del entrevistador, en el primer ejemplo, es posible apreciar el énfasis que pone en hacer pensar a la participante cómo su vida sería diferente con su papá. Se aprecia que esto genera reflexión y ciertas complicaciones, ya que es muy probable que el recuerdo de su padre sea bastante vago. En el segundo, el entrevistador usa cierto «asombro» de cómo se sigue tomando en cuenta al padre, y la respuesta del adolescente denota aceptación de la dificultad del proceso, pero al final lo califica como algo «necesario».

Entonces ¿qué es lo que pierde? Se puede llegar a considerar que lo importante es el hecho de que se ha mantenido la presencia del padre (en lo simbólico y en lo funcional), y en estos casos en particular, reconocen la importancia no solo para ellos, sino también para su madre. Tiene que ver también con el apoyo emocional que un hijo puede proveer a una madre en un proceso de separación en estas condiciones, referido Jurkovic, Kuperminc, Perilla, Murphy, Ibáñez y Casey (2004).

Reconocer lo que hace falta también puede considerarse un factor importante de afrontamiento en este proceso. Consideramos que tampoco sería esperado que las personas no se dieran cuenta de lo que se genera (sentimientos de tristeza, por ejemplo) por el hecho de que el padre no esté físicamente en su vida cotidiana. No obstante, parece que tiene que ver con un proceso de acoplamiento a las condiciones en las que es más factible enfrentar esta separación. Como ha sido señalado por Falicov (2007) uno de los aspectos psicosociales que se generan a consecuencia de los procesos de migración es que produce familias trasnacionales, es decir, que a pesar de las fronteras que separan a uno o varios miembros de la familia, siguen unidos a través de la distancia; sin embargo, debido a estos cambios territoriales, estas familias pueden llegar a experimentar procesos importantes y/o significativos de estrés relacional.

El segundo núcleo temático, denominado apoyo/ reacoplamiento de la familia extensa ante la ausencia paterna, se relaciona con las reacciones que la familia extensa (tanto política como consanguínea) tiene con la mamá y los hijos del padre migrante cuando este se ausenta. Tiene que ver con el establecimiento de una frontera lo suficientemente clara para que la familia nuclear no se subsuma en la extensa, y lo suficientemente permeable como para que la familia extensa y la familia nuclear se puedan apoyar mutuamente en este proceso. Veamos por ejemplo el siguiente testimonio:

\section{E1.- ¿Cuánto tiempo tenia $\mathrm{N}$ ?}

Mamá.- Como 5,6 meses ... la $\mathrm{N}$ la más chiquita y le dije, deja le digo a mi mamá, y mi mamá se agarró llorando, no pos me la dejas ni modo y que sabe qué y fue que mi papá me dijo, anda, mi papá es muy palabrero, me dijo anda vas a andar como las chingadas húngaras, no apá, «es que aquí no vas a hacer como aquí en tu casa, ahí te vas a estar cambiando cada tres meses, cada mes y luego si tú tienes un patrón vas a trabajar ya y él un rato y tu otro y es un cuartito de 2 x 3 metros»...

Como se pudo observar en el párrafo anterior, la reacción de la familia extensa de la madre presentó cierta ambivalencia, por un lado, la mamá muestra una actitud solidaria con la ayuda hacia la nieta, mientras que el papá expresa su «malestar» por la modificación en la estructura familiar que produce la migración del padre. Sin embargo, este mismo papá se muestra solidario en otra parte del relato de la persona:

Mamá: hubo una ocasión en que a mi hija L le agarró el pie mi sobrina en la bicicleta, mi cuñado, el hermano de mi esposo vivía en la esquina, él es el más grande y mi esposo el más chico, el talón lo traía colgado, de aquí y ya llega mi cuñado con mi hija ahí alzada y le colgaba su taloncito, y su talón así, su carne y yo la llevo al hospital ah y mi cuñado me dice oye no te puedo llevar porque mi camioneta no tiene placas y no traigo la cartera y mi mamá es bien palabrera cuando se requiere, no que pues y mi papá, ahora le van a quitar un riñón, el está enfermo, estaba tembloroso, como nervioso y mi hija L es la 
más chiqueada de la casa, ahí entonces mi papa se sintió mal, pos ahí le veía el talón, ella estaba chiquitita y flaquita y ya ahí vamos como era sábado, no te miento mi papá iba temblando, no se paró en los semáforos ni nada, llegamos yo iba temblando y mi papá yo, yo lloré y lloré.

Como se puede ver en este ejemplo, la función que ejerza la familia extensa puede convertirse en un gran factor de apoyo tanto para lo instrumental como para lo emocional. Como ya ha sido descrito anteriormente, el papel del hombre en las culturas latinas sigue estando muy asociado a la ayuda y a la protección (Kanaiapuni, 2000). Resulta lógico entender que ante la ausencia del papá que ha emigrado, los varones que quedan en la familia extensa, se sientan con cierto compromiso de ayudar en esos roles tradicionalmente masculinos. Es probable que esto también tenga algo que ver con los resultados encontrados por Abrego (2006) en el sentido de que el padre emigrado es hasta cierto punto más «reemplazable» que la madre que decidiera emigrar.

Por último, el núcleo temático número 3, comunicación continua y significativa del padre con su familia, se refiere a la comunicación que tanto el padre como la familia generan durante los períodos de ausencia, que se vuelve un mecanismo eficaz para afrontar la separación.

Obsérvese por ejemplo la reacción de los hijos en el momento en que ya tienen línea telefónica:

Mamá.- Eh y yo casi no hablaba con él, los primeros seis meses casi no hablaba, no teníamos teléfono y en mi casa, mi mamá le daba más preferencia a mi cuñada que a mí, o sea yo hablaba los miércoles, los sábados y los domingos y duraba una hora, porque el teléfono no era de nosotros, hasta que ya metí trámites a TELMEX y tuve mi teléfono, y cuando tuve mi teléfono mis niños ya hablaban cada rato, ya no querían salir, ya ni R quería ir al cine, «no es que va a hablar mami, mi papá va a hablar», y ya entonces mi mamá decía, mi mamá era muy dura, me decía, «ay ya, es mucha payasada que hable tanto con los niños, qué tanto hablas?» Me decía mi mamá, hay mamá pos que tiene lo que hablé, cuando mi papá estaba en el Norte a mi mamá le mandaba sus cartas ino?, porque mi mamá todavía tiene sus cartas de cuando mi papá se las mandaba, y ya pues de la forma esa me fui haciendo más...

(Entrevista realizada el 25 de enero de 2010).

Como se observa en la viñeta pasada, la mamá entiende la importancia de la comunicación significativa, aun cuando a los ojos de la familia extensa pudiera parecer exagerada la reacción de los hijos. Por otro lado, éstas llamadas también representan la opción de un intercambio cultural entre el país de origen y el país de emigración del padre:

E:- Y del 2004 a la fecha son 6 años

¿cómo han estado las cosas con tu papá?

S.- Pues ahorita desde que se fue no nos

ha dejado de hablar, siempre nos habla diario para desearnos las buenas noches o saludarnos... yo platico mucho con él cuando habla.

E.-¿Y de qué platican?

S.- Pues me pregunta cómo voy aquí o...

si no he tenido problemas así, o... el me está enseñando a hablar en inglés, él va allá a clases de inglés para aprender y pues él me está enseñando.

Como se puede apreciar, el medio de la llamada, en el relato de esta persona, se ha vuelto una forma de enseñanza de lo que el papá está aprendiendo en el país de emigración. Es una «conexión» entre papá e hija en todo el sentido de la palabra. La idea de familia transnacional queda ilustrada en este ejemplo, así como la importancia que pueden tener las tecnologías de la información en estos procesos. Como ha sido señalado por diversos autores como Gergen (1991) en el posmodernismo las tecnologías de la información han permitido que las distancias se acorten, y que existan no solo realidades caraa-cara, sino también realidades virtuales. Al parecer, este paradigma comunicativo también ha revolucionado las relaciones en familias con padre emigrante a los EUA.

\section{Discusión}

El presente estudio ha abordado el proceso de migración de familias mexicanas a Estados Unidos en la dimensión de separación familiar que conlleva en muchos de los casos. Consideramos que uno de los principales atributos del presente estudio es que se enfoca a los familiares que se «quedan» en el país de origen, ya que mucha de la literatura existente sobre el tema ha investigado más bien a los migrantes en el país de destino, como 
lo reflejan los artículos seminales de Suárez-Orozco, Todorova y Louie (2002), Bacigalupe y Lambe (2011) o Falicov (2007).

Por otro lado, otra de las características relevantes de este trabajo es que intenta describir el proceso de afrontamiento en términos del sistema familiar, más que desde el punto de vista del individuo. Los estudios sobre estrés, de manera general, se han enfocado más en el afrontamiento del individuo y sus recursos, y menos en términos que podríamos denominar «relacionales».

De este estudio cualitativo se infiere que la ambigüedad de la separación paterna producto de la emigración parece ser la característica más relevante dentro de tal proceso. La información recolectada muestra que los miembros de la familia que se quedan en el país de origen ven en la ambigüedad (estar/no estar) el principal factor a afrontar, de acuerdo a la terminología utilizada por Boss (2001). Esta ambigüedad parece estar centrada en que en las familias entrevistadas parece que la migración es vista como un paso necesario para la sobrevivencia de la familia, aún a pesar de la separación paterna que puede llegar a durar incluso hasta años.

El abordaje familiar con el que se llevó a cabo este estudio, siguiendo la teoría estructural de Minuchin (1974) parece indicar que las familias de origen juegan un papel muy importante durante este proceso de afrontamiento, ya que proporcionan soporte tanto instrumental como emocional sobre todo cuando la madre ha quedado a cargo de los hijos. De hecho, la teoría de la parentalización/responsabilidad filial (Boszormenyi-Nagy, 1973; Jurkovic, Kuperminc, Perilla, Murphy, Ibañez \& Casey, 2004). Tiene mayor utilidad en el estudio de familias completas emigradas, y no en familias separadas por la emigración, donde parece que hay que incluir más a las familias extensas para un mejor entendimiento del fenómeno. Aquí es posible inferir que teorías como la estructural de Minuchin (1974) en su versión terapéutica ofrecen esquemas un tanto «estrechos» para entender el funcionamiento de estas familias con padre emigrante, ya que los límites y fronteras son constantemente negociados entre la familia de origen y la extensa. Dicho de otra forma, el entendimiento de las jerarquías, los funciones y roles de las familias con padre migrante, necesita de una flexibilidad con respecto de las formas tradicionales observadas en familias latinas, aunque al mismo tiempo, es posible observar cómo esta tradición prevalece aún con la distancia. Así, una teoría como la estructural de Minuchin proporciona un buen mapa inicial que es necesario actualizar para poder ser usado en los fenómenos migratorios. Por lo demás, en este estudio se observa en general que la autoridad del padre se trata de conservar, aun cuando sus funciones tienen que negociarse constantemente de acuerdo a la circunstancia.

Conceptos como el de familias transnacionales parecen tener una gran relevancia en el futuro, donde cada vez será más frecuente el fenómeno de la migración junto con el sostenimiento de los lazos familiares. En este sentido, en la presente investigación se encontró que los mecanismos de afrontamiento familiar que mostraron mayor eficacia están asociados a actos simbólicos que les permiten sentirse conectados en la separación. La hora de la llamada, la esperanza de un reencuentro y el mensaje de texto diario parecen ser cuasi-rituales que permiten que el padre y la familia en el país de origen sigan sintiendo una conexión importante. En este sentido, las tecnologías de la información están jugando un papel trascendental, como en muchos otros aspectos de la vida mundial, para mantener de manera virtual los lazos de las familias separadas por la migración. Tal vez futuros estudios pudieran enfocarse de manera más directa y cuantitativa a la investigación del impacto de las tecnologías de la información en el afrontamiento de los procesos de separación familiar producto de la migración. Una intervención muy lógica, en este sentido, con la población masculina que emigra a los Estados Unidos sería entrenarlos en el manejo de la comunicación virtual como un recurso indispensable para mantener el contacto con su familia. Por otro lado, uno de los usos de estas tecnologías para futuras investigaciones en el tema, pudiera ser emplearlas en la misma investigación, de manera tal que pudieran llevarse a cabo entrevistas vía chat con el padre ausente, y así se pudiera tener una visión más completa de la familia, cambiando el esquema de tener que entrevistar de un lado u otro de la frontera. Sin duda que los cambios traídos por el transnacionalismo y la globalización de las familias también pudieran tener un impacto en la forma en que se les investiga, aun cuando esto pudiera implicar un rompimiento con la tradición vis-a-vis o cara a cara de una herramienta como la entrevista cualitativa. 


\section{Referencias}

Abrego, L. (2006). Growing up With Hopes of Leaving: How Transnationalism Affects Non-Migrant Youth. Conferencia presentada en la Reunión de la Asociación de Estudios Latinoamericanos 2006.

Bacigalupe, G. \& Lambe, S. (2011). Virtualizing Intimacy: Information Communication Technologies and Transnational Families in Therapy. Family Process, $50,1,12-26$.

Becvar, D. S. \& Becvar, R. J. (1996). The structural Approach. Family Therapy a Systemic Integration (pp. 187-206). Needham Heights, MA: Allyn \& Bacon.

Bolívar, A. Domingo, J. \& Fernández, M. (2001). Fundamentos filosóficos y epistemológicos de la narratividad en la investigación biográfico-narrativa en educación. Enfoque y metodología. Madrid: La Muralla.

Boss, P. (2001). La pérdida ambigua. Como aprender a vivir con un duelo no terminado. Barcelona: Gedisa.

Boszormeny-Nagy, I. \& Spark, M. G. (1973). Lealtades Invisibles. Buenos Aires: Amorrortu.

Falicov, C.J. (2007) Working With Transnational Immigrants: Expanding Meanings of Family, Community, and Culture. Family Process, 46(2), 157-171.

Gergen, K. (1991). El yo saturado: dilemas de identidad en el mundo contemporáneo. Barcelona: Paidós.

Hernández, R., Fernández, C. \& Baptista, P. (2006). Metodología de la Investigación. México: McGraw-Hill.

Husserl, E. (1991). La crisis de las ciencias europeas y la fenomenología trascendental. Barcelona: Crítica.

Instituto Nacional de Estadística \& Geografía e Informática. (2004). Población emigrante con destino a los Estados Unidos de América por período según sexo, 1990 a 1995, y 1995 a 2000. Recuperado el 16 de enero de 2008 de www.inegi.gob.mx/est/contenidos/espa$\mathrm{nol} / \mathrm{rutinas} / \mathrm{ept} \cdot \mathrm{asp} ? \mathrm{t}=\mathrm{mpob} 66 \& \mathrm{~s}=\mathrm{est} \& \mathrm{c}=3243$
Jurkovic, G., Kuperminc, G., Perilla, J., Murphy, A., Ibáñez, G. \& Casey, S. (2004). Ecological and Ethical Perspectives on Filial Responsibility: Implications for Primary Prevention with Immigrant Latino Adolescents. The Journal of Primary Prevention, 25(1), 81-104.

Kanaiapuni, S. M. (2000). Reframing the Migration Question: an Analysis of Men, Women and Gender in Mexico. Social Forces, 78(4), 1311-1348.

Lazarus, R. S. (1991). Psychological Stress in the Workplace. Journal of Social Behavior and Personality, 6, $1-13$.

Lazarus, R. S. \& Folkman,S. (1984). Stress, Appraisal and Coping. New York: Springer.

Matheny, K. B. \& McCarthy, C. J. (2000). Write Your Own Prescription For Stress. Oakland, CA: New Harbinger Publications, Inc.

Minuchin, S. (1974). Families and Family Therapy. London: Harvard University Press.

Orozco, J. C. \& Sarabia, E. (2005). Dejan México 485 mil al año [Versión electrónica], Mural, Negocios, 1. Recuperado el 3 de octubre de 2006 de http://0proquest.umi.com.millenium.itesm.mx:80/pqdli nk did $=840145401 \&$ sid $=1 \&$ Fmt $=3 \&$ clientId $=23693 \& \mathrm{RQT}=309 \& \mathrm{VName}=\mathrm{PQD} *$

Patton, M. (1980). Qualitative Evaluation Methods. Beverly Hills, CA: Sage.

Roque, B. E., Kuperminc, G.P., Ramírez, M. G., \& Álvarez, A. (2009). El sueño americano: la experiencia de familias mexicanas emigrantes al sureste de los Estados Unidos. Enseñanza e Investigación en Psicología, 14 (2), 339-354.

Suárez-Orozco, C., Todorova I. \& Louie J. (2002). Making up for Lost Time: The Experience of Separation and Reunification Among Immigrant Families. Family Process, 41(4), 625-643. 